\title{
The Straight Talk for Infant Safe Sleep Program in Support of American Academy of Pediatrics Safe Sleep Guidelines
}

Barb Himes, IBCLC

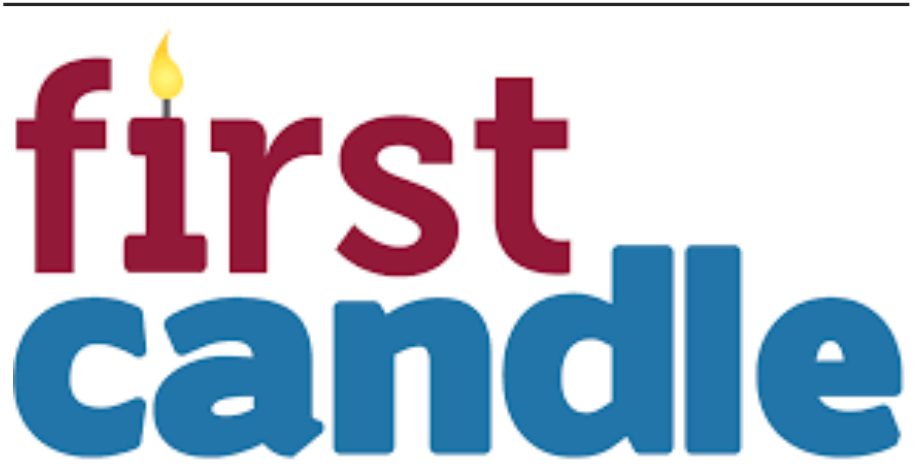

Saving babies. Supporting families.

First Candle's efforts to support families during their most difficult times and provide new answers to help other families avoid the tragedy of the loss of their baby are without parallel.

First Candle is a longstanding member of the resource pool available to families and the postpartum health care community, but there are also others - Title $\mathrm{V}$ partners -- that could have a place on the resource list for neonatology and perinatology professionals.

"First Candle is a longstanding member of the resource pool available to families and the postpartum health care community, but there are also others - Title V partners -- that could have a place on the resource list for neonatology and perinatology professionals."

Our work focuses on reducing infant mortality through our Straight
Talk for Infant Safe Sleep program, a constructive and collaborative training for health care professionals that enables them to help families understand the importance of and adopt the infant Safe Sleep Guidelines last updated by the American Academy of Pediatrics (AAP) in 2016. We also provide bereavement support for families coping with the loss of an infant.

\section{Continuous Improvement, Title V and National Performance Measures}

We recently took part in a webinar series hosted by the Maternal and Child Health Bureau (MCHB) of the Health Resources and Services Administration (HRSA), designed to help Maternal and Child Health divisions in state and jurisdiction health departments improve services and make evidence-based decisions when developing their five-year state action plans. The subject matter expert-led sessions were designed to help Title $\mathrm{V}$ program staff identify evidence-based strategies that address the 15 National Performance Measures (NPMs) that focus on public health and are relevant to Title V (The Maternal and Child Health Block Grant Program) services.

\section{Selected Areas of Focus}

Our particular focus during this series of sessions was on NPMs around breastfeeding. The AAP recommends that all infants (including premature and sick newborns) exclusively breastfeed for about six months, as human milk supports optimal growth and development by providing all required nutrients during that time. Breastfeeding strengthens the immune system, improves normal immune response to certain vaccines, offers possible protection from allergies, and reduces the probability of SIDS.

Breastfeeding has been shown to extend benefits to the mother, including improved confidence and bonding with the baby and a reduction in anxiety and postnatal depression, as well as increased release of oxytocin while breastfeeding, leading to a reduction in postpartum hemorrhage and quicker return to a normalsized uterus over time. There are also indications the mother may be less likely to develop breast, uterine, and ovarian cancer, and have a reduced risk of osteoporosis. (1)

Research indicates, however, that there is a gap between the percentage of infants who are ever breastfed and those breastfed exclusively through six months $(83.2 \%$ ever breastfed; $24 \%$ exclusively for six months) (2). We were able to suggest proven strategies that health departments can implement to help improve breastfeeding outcomes, such as lactation consultants and fatherhood engagement programs. Also worth noting was the full range of NPMs covered by the MCHB sessions, including:

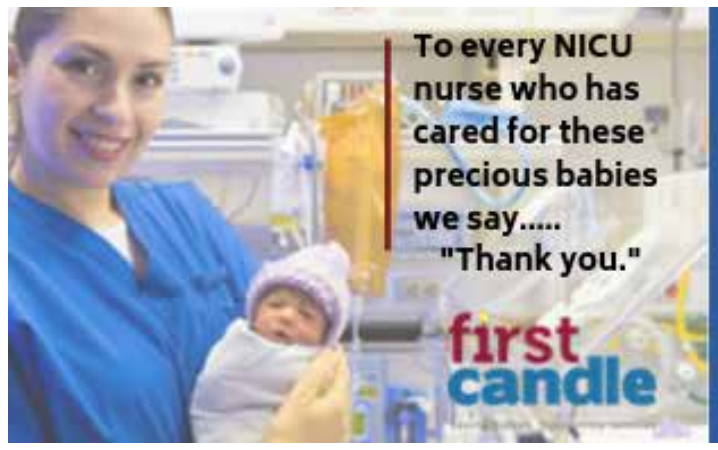

\section{Did you know that premature and low birth weight babies have a $4 \times$ greater risk for SIDS?}

At First Candle we're educating parents, grandparents and caregivers about safer sleep to make sure all babies reach their first birthday. Learn more at firstcandle.org 
NPM 1: Well-Woman Visit

NPM 2: Low-Risk Cesarean

NPM 3: Perinatal Regionalization

NPM 4: Breastfeeding

NPM 5: Safe Sleep

NPM 6: Developmental Screening

NPM 7: Child Safety/Injury

NPM 8: Physical Activity

NPM 9: Bullying

NPM 10: Adolescent Well-Visit

NPM 11: Medical Home

NPM 12: Health Care Transition

NPM 13: Oral Health

NPM 14: Smoking

NPM 15: Adequate Insurance Coverage

\section{"The goal of regional perinatal care, for} example, is to ensure that mothers and newborns at the highest risk for morbidity and mortality deliver at birthing facilities equipped with appropriate personnel, resources, and capabilities to meet their needs. "

The goal of regional perinatal care, for example, is to ensure that mothers and newborns at the highest risk for morbidity and mortality deliver at birthing facilities equipped with appropriate personnel, resources, and capabilities to meet their needs. Proven strategies in addressing this include adoption of standard definitions for hospital level of care and statewide assessment of personnel, resources, and capabilities of birthing facilities.

In this area, Title $\mathrm{V}$ agencies can design, fund, and manage the implementation of perinatal systems in partnership with hospitals and perinatal professionals, and fund and manage the implementation of maternity facility assessment in partnership with hospitals and perinatal professionals.

With regard to safe sleep, strategies presented at the session included implementing a multicomponent strategy (systems approach) that targets caregivers, childcare providers, health care providers, and hospital systems, and providing training to professionals who interact with expecting and new mothers and families that emphasizes nuanced approaches, taking into account family needs, beliefs, and context.

In these areas, Title $\mathrm{V}$ agencies can build systems by engaging appropriate programs and partners across functions (such as hospitals, healthcare provider organizations, community-based programs, etc.) and utilizing train-the-trainer programs.

\section{Awareness of Available Resources}

These examples serve as a reminder of the range of disciplines intrinsic to Title $V$ agencies, which could be a part of the prenatal, perinatal, and postpartum health care professional's inventory of support services.

It may be worthwhile to identify resources at the community, health department, and hospital-level that can be available to provide support for parents of newborns. This new era of novel coronavirus presents us with a renewed commitment to maintaining effective and high-quality levels of care, while at the same time facing known and as yet-unfolding challenges.

At such a time, being aware of what resources are available and partnering to take advantage of areas of expertise can be effective strategies toward continued excellence in maternal and infant health care.

\section{References:}

1. American Academy of Pediatrics Section on Breastfeeding. Breastfeeding and the use of human milk. Pediatrics. 2012 Mar;129(3): e827-41. https://pediatrics.aappublications.org/content/129/3/e827

2. Trends in SUID by Cause, 1990-2017 from CDC's SUID and SIDS Data and Statistics portal. https://www.cdc.gov/sids/data. $\underline{h t m}$

Disclosure: The author is the Director of Education and Bereavement Services of First Candle, Inc., a Connecticut not for profit 501c3 corporation.

\section{NT}

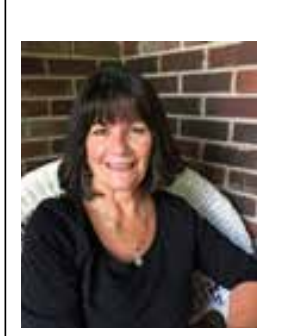

Barb Himes, IBCLC

Director of Education and Bereavement Services

First Candle

49 Locust Avenue, Suite 104

New Canaan CT 06840

Telephone: 1-203-966-1300

For Grief Support: 1-800-221-7437

barb@firstcandle.org

www.firstcandle.org

\section{Time is precious, just like your patients.}

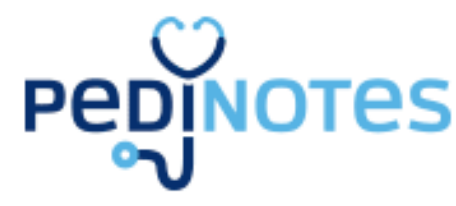

